controls with a mean age of $42.12 \pm 7.22$ mean BMl of $25.87 \pm 3.51$ with an unsignificant difference between two groups.

Serum sclerostin level significantly higher in PsA patients compared to controls with a mean of $(0.64$ and $0.37 \mathrm{ng} / \mathrm{ml})$ respectively, positive significant correlation with patients' age, disease activity scores, ultrasonographic findings of inflammation and damage at the enthesis as well as negative correlation with DEXA at lumbar spine. A positive though non-significant correlation detected between serum sclerostin and Leeds clinical enthesitis index (LEI) and CRP.

Conclusions: sclerostin plays important role in pathogenesis of psoriatic arthritis and associated with bone damage either systemic or localized. Further studies for the effect of treatment on serum sclerostin, ultrasonographic and bone mineral density findings is recommended

References:

[1] Homaira Rahimi \& Christopher T. Ritchlin. Altered Bone Biology in Psoriatic Arthritis. Curr Rheumatol Rep (2012) 14:349-357.

[2] Matzelle MM, Gallant M, Condon KW, et al. Resolution of inflammation induces osteoblast function and regulates the Wnt signaling pathway. Arthritis Rheum 2012; 64:1540-1550.

[3] Healy PJ and Helliwell PS: "Measuring clinical enthesitis in psoriatic arthritis: assessment of existing measures anddevelopment of an instrument specific to psoriatic arthritis." Arthritis Care and Research 2008; 59(5):686-691.

[4] Garrett S, Jenkinson T, Kennedy LG et al. A new approach to defining disease status in ankylosing spondylitis: the Bath ankylosing spondylitis disease activity index. J Rheumatol (1994); 21 (12): 2286-2291.

[5] Ibrahim G, Groves C, Chandramohan M, Beltran A, Valle R, Reyes B, Healy $P$, Harrison A and Helliwell P S. Clinical and Ultrasound Examination of the Leeds Enthesitis Index in Psoriatic Arthritis and Rheumatoid Arthritis. ISRN Rheumatology Volume 2011(2011), Article ID 731917, http://dx.doi.org/10.5402/2011/731917.

Disclosure of Interest: None declared

DOI: 10.1136/annrheumdis-2017-eular.1317

\section{AB0788 DIFFERENCES IN THE PHENOTYPIC EXPRESSION OF RECENT ONSET PSORIATIC ARTHRITIS AMONG MEN AND WOMEN BASELINE DATA FROM THE REAPSER STUDY}

R. Queiro, A. Laiz Alonso, H.S. Park, C. Montilla Morales, E. Galíndez Agirregoikoa, J.J. Bethencourt Baute, S. Bustabad Reyes, P. Tejón Menéndez, M.Á. Belmonte, J.A. Pinto Tasende, E. Alonso Blanco Morales, J. Ramír. Rheumatology, Hospital Universitario Central de Asturias (Coordinating Center), Oviedo, Spain

Background: The natural history of psoriatic arthritis (PSA) according to gender of patients is an aspect of the disease only partially studied. In prospective PsA cohorts such information is barely known.

Objectives: To analyze baseline gender differences in the REAPSER cohort (Psoriatic Arthritis Registry of the Spanish Society of Rheumatology).

Methods: Observational, multicenter study (34 centers), with consecutive inclusion. We included adults of both sexes 18 years of age or older with PsA that met CASPAR criteria, and duration of less than two years since the appearance of symptoms attributed to PsA. Annual follow-up visits will be carried out for 5 years. Measurements: socio-demographic data; employment status and impact of the disease; family history of PsA and other inflammatory diseases; comorbidities and treatment; lifestyle; use of health services; clinical status at the time of diagnosis of PsA; anthropometric data; clinical evaluation of PsA manifestations; radiographic

\begin{tabular}{lccc}
\hline Parameter & Men $(\mathrm{N}=142)$ & Women $(\mathrm{N}=68)$ & P values \\
\hline Age (years) & $50.2(13.8)$ & $49.6(14.1)$ & $\mathrm{NS}$ \\
University studies & $18.3 \%$ & $16.2 \%$ & $\mathrm{NS}$ \\
Active worker & $65 \%$ & $49.3 \%$ & $<0.001$ \\
BMI & $27.3(4.8)$ & $28.5(6)$ & $\mathrm{NS}$ \\
Waist-hip index & $0.94(0.1)$ & $0.87(0.1)$ & $<0.001$ \\
Smoker & $29.6 \%$ & $32.4 \%$ & $\mathrm{NS}$ \\
Alcohol consumption & $45.1 \%$ & $16.2 \%$ & $<0.001$ \\
Psoriasis family history & $40.1 \%$ & $45.6 \%$ & $\mathrm{NS}$ \\
PsA family history & $8.5 \%$ & $10.3 \%$ & $\mathrm{NS}$ \\
Charlson's Cl (>3) & $18.3 \%$ & $17.6 \%$ & $\mathrm{NS}$ \\
Depression & $7.7 \%$ & $19.4 \%$ & 0.014 \\
Common Psoriasis & $81 \%$ & $61.8 \%$ & 0.003 \\
Pustular Psoriasis & $2.1 \%$ & $11.8 \%$ & 0.006 \\
Onicopathy & $59 \%$ & $49 \%$ & $\mathrm{NS}$ \\
PASI & $1.5(0.6-4.4)$ & $1.2(0.6-3)$ & $\mathrm{NS}$ \\
Peripheral pattern & $80.3 \%$ & $83.8 \%$ & $\mathrm{NS}$ \\
Axial pattern & $7 \%$ & $1.5 \%$ & $\mathrm{NS}$ \\
Mixed Pattern & $12.7 \%$ & $14.7 \%$ & $\mathrm{NS}$ \\
BASDAI (0-10) & $4.1(2.2-6)$ & $4.8(2.4-7.3)$ & 0.008 \\
BASFI (0-10) & $1.8(0.4-4.5)$ & $2.9(1.4-4.7)$ & 0.065 \\
Dactylitis & $31.4 \%$ & $47.1 \%$ & 0.028 \\
Pain (0-10) & $4.5(2-7)$ & $6(4-7.5)$ & 0.021 \\
PGA (0-10) & $5(3-7)$ & $6(3.5-8)$ & 0.042 \\
ESR (mm/h) & $12(5-20)$ & $21(10-29.5)$ & 0.001 \\
\hline CI: comorbidity & & & \\
\hline
\end{tabular}

Cl: comorbidity index. PASI: Psoriasis Area and Severity Index. PGA: Patient's Global Assessment. Data are expressed in percentages, means with SD (Standard Deviation), medians and IQR (Interquartile Range). There were no significant differences in SJC, TJC or PsAID (Psoriatic Arthritis Impact of Disease) evaluation; analytical determinations; treatment of PsA. The study has been approved by the Ethical committees of the participating centers. Comparative statistical analysis: for qualitative variables, the $\chi$-square or the Fisher exact statistic were used. For non-normal quantitative variables, non-parametric tests were used and for normal quantitative variables, Student's t-test was used.

Results: The results are expressed in the table.

Conclusions: The baseline data from this prospective cohort point to significant differences in the phenotypic expression of PsA between men and women. Thus, in women, the prevalence of dactylitis and pustular psoriasis was higher, there were higher rates of depression and a perception of higher disease activity. Women scored higher on the pain linked to their arthritis and the activity of axial disease. They also had biological activity parameters (ESR) higher than that of men. It is necessary to determine if these differences are maintained or change over time. Disclosure of Interest: None declared

DOI: 10.1136/annrheumdis-2017-eular.5039

\section{AB0789 THE PSORIATIC ARTHRITIS PATIENT'S JOURNEY: SPECIAL EMPHASIS ON DIAGNOSIS AND TREATMENT DELAYS}

S. Moyano, M. Brom, F.B. Mollerach, L.E. Pompermayer, M.L. Acosta Felquer, M. Scolnik, J. Marin, L.G. Ferreyra Garrott, L.J. Catoggio, J.E. Rosa,

E.R. Soriano. Rheumatology, Hospital Italiano de Buenos Aires, Buenos Aires, Argentina

Background: A delay in diagnosis and treatment of Psoriatic Arthritis (PsA) is associated with increased disability and damage in the long term. There is currently scarce data available about diagnosis delay, referrals delays, and time to first treatment in patients with PSA in developing countries.

Objectives: To describe the journey of patients with psoriatic arthritis, with special emphasis on diagnosis and treatment delays.

Methods: All patients with PsA registered in the Rheumatology Unit data base (between 2000-2016), with complete data, were included. Electronic medical records were manually revised, and the following data were obtained: date of first visit to a Dermatologists due to Psoriasis ( $\mathrm{PsO}$ ) symptoms, date of $\mathrm{PsO}$ diagnosis, date and type of first musculoskeletal symptom, specialty of physician seen at first visit for musculoskeletal symptoms, date of PsA diagnosis, date and reason for prescription of first Disease Modifying anti-Rheumatic Drug (DMARD) Primary outcome variable was mean lag time between first musculoskeletal symptom and diagnosis of PsA. Other variables calculated were: mean lag time between first musculoskeletal symptom and first physician encounter because of those symptoms, mean lag time to first DMARD and mean lag time between $\mathrm{PsO}$ diagnosis and PsA diagnosis. Variables associated with a delay in PsA diagnosis (more than one year delay) were analyzed in multivariable analysis (logistic regression).

Results: 93 patients were included, mean age 60.8 years (SD: 15.3 ), 61\% males. Mean age at time of PsA diagnosis was 52 years (SD: 14.8). The most common musculoskeletal symptom was arthralgia (46\%), followed by arthritis $(37 \%)$, enthesitis $(6 \%)$, low back pain $(6 \%)$, and dactylitis $(4 \%)$. Mean lag time between first musculoskeletal symptoms and visit to a physician because of those symptoms was 16,8 months (SD: 44,4) (median: 1,92 (IQR: 0,35-11,6). In Only $33 \%$ of the cases the first specialist seen was a Rheumatologist. Mean lag time between first musculoskeletal symptom and diagnosis of PsA was 19,2 (SD: 28,8) months (Median: 7,2 (IQR: 2,4-21,6 months). In 90 patients (97\%), the diagnosis of $\mathrm{PsO}$ preceded the diagnosis of PsA, a mean time of 15.1 years (SD: 14.4). 83 patients (89\%) received traditional DMARDs, $82 \%$ because of the musculoskeletal symptoms, with a mean lag time between PsA diagnosis and initiation of DMARDs of 11,4 months (SD: 31,2$)$ (Median: 0,48 (IQR: 0-4,3) months). Forty-three patients (46.2\%) had a delay on PsA diagnosis equal or greater than 1 year. In logistic regression analysis, including age, sex, first specialist seen and type of musculoskeletal symptom, none was independently associated with a delay equal or greater than 1 year in PsA diagnosis.

Conclusions: Mean time between symptoms' onset and PsA diagnosis was relatively short. However, a delay greater that one year was observed in almost half of patients. As none of the variables studied was associated with a delay in diagnosis, more studies are needed to identify potential actions that would help reducing this delay.

References:

[1] Kane D, et al. Rheumatology 2003;42:1469-1476.

[2] Haroon M, et al. Ann Rheum Dis 2014:0:1-6.

[3] Gladman DD, et al. Arthritis Rheum 1998;41:1103-1110.

Disclosure of Interest: None declared

DOI: 10.1136/annrheumdis-2017-eular.2231

\section{AB0790 HYPERURICEMIA IN PSORIATIC ARTHRITIS: PREVALENCE AND ASSOCIATED FACTORS}

T. Gudu, A. Peltea, A. Balanescu, V. Bojinca, D. Opris, D. Predeteanu, R. Ionescu. Rheumatology, Sf Maria Hospital, UMF Carol Davila, Bucharest, Bucharest, Romania

Background: Hyperuricemia is frequent in psoriatic arthritis (PsA) and it seems to be related to metabolic syndrome rather than to extensive psoriatic skin disease [1]. 